\title{
Experience Since MELD Implementation: How Does the New System Deliver?
}

\author{
Markus Quante, Christoph Benckert, Armin Thelen, and Sven Jonas \\ Department of Visceral, Transplantation, Thoracic and Vascular Surgery, University Hospital Leipzig, Liebigstr. 20, \\ 04103 Leipzig, Germany \\ Correspondence should be addressed to Christoph Benckert, Christoph.Benckert@medizin.uni-leipzig.de
}

Received 17 January 2012; Revised 3 September 2012; Accepted 3 September 2012

Academic Editor: Pierluigi Toniutto

Copyright ( 2012 Markus Quante et al. This is an open access article distributed under the Creative Commons Attribution License, which permits unrestricted use, distribution, and reproduction in any medium, provided the original work is properly cited.

\begin{abstract}
Because of increasing waiting-list mortality, the MELD (Model for End-Stage Liver Disease) allocation system was implemented within most countries of the Eurotransplant area on December 16, 2006. Five years have now passed, and we review in this paper the effects of the MELD-based allocation upon the waiting list for liver transplantation, on peri-operative management and on postoperative outcome. Giving priority to sicker patients on the waiting list has resulted in a significant increase in mean MELD score at the time of organ allocation. Consequently, there has also been a significant reduction in waiting-list mortality. However, in Germany a worsening in postoperative outcome, mainly in the group of high-MELD recipients ( $\geq 30$ points), has been reported. This paper presents comprehensive results following liver transplantation within the MELD era. Especially for the group of highrisk recipients, risk factors for impaired survival are presented and discussed.
\end{abstract}

\section{Background}

The last two decades have seen a steadily increasing number of patients on the waiting list for solid-organ transplantation. In the context of continual donor-organ shortage, this has engendered a persistent problem of allocation. As not every patient on the waiting list will be able to have timely organ access, the question of whom to serve first remains acute. Therefore, the existence of mandatory allocation rules is indispensable. When the German transplant law was enacted in 1997, the allocation system was based equally on cumulative waiting time and on individual clinical condition, as reflected by the Child-Turcotte-Pugh (CTP) score. This allocation principle was intended to serve the central demand for best utilization of each donor organ, in the sense of providing an optimum balance between medical urgency and postoperative outcome. However, throughout the years, waiting time became a major discriminating factor for patients on the waiting list; patients with acute liver decompensation, who were in urgent need of a transplantation but who had not accumulated enough waiting time, had a high risk of dying while on the waiting list, without receiving an organ offer. In addition, since 2002 there was an accelerated increase in numbers of patients on the waiting list for liver transplantation. These two factors led to a significant increase in waiting-list mortality [1].

Finally, on 16 December 2006 the MELD score was implemented as the basis for new allocation system in many countries within the Eurotransplant area. This model provides a prediction of 3-month mortality without liver transplantation. The score is calculated by combining bilirubin, creatinine, and INR (international normalised ratio) values only, and it therefore has an objective basis [2]. However, as there are many different possible underlying diseases that, despite chronic liver decompensation, often have only a modest impact on laboratory results, standard exceptions to the MELD system, with adjustment of the score, have been defined. For example, patients suffering from hepatocellular carcinoma are given an adjustment in their MELD score because of the underlying malignancy and the consequent anticipated tumour growth during the waiting period-risk factors that may not be reflected by laboratory results. Further common MELD exceptions are polycystic liver disease, cystic fibrosis, and metabolic 
disorders. In 2008-after first review and adaptation of the allocation system-also cholestatic liver diseases have been added to the standard exceptions due to the low impact of laboratory MELD in these patients. But the MELD exception for cholestatic liver diseases is only fulfilled in selected cases with septic complications. All diseases representing a standard exception according to the current guidelines defined by the German Medical Association and their initial adjusted MELD score is depicted in Table 1 [3]. The MELD-based allocation was intended to reduce waiting-list mortality by giving priority to cases with medical urgency. Five years after its implementation in the Eurotransplant area, there have been significant effects on waiting-list development, perioperative management and postoperative outcome; these are presented and discussed in this paper.

\section{Waiting-List Mortality}

The implementation of the MELD allocation system has reflected a change towards an urgency-oriented model that was intended to improve organ access for sicker patients with higher MELD scores. At our centre, there was a significant reduction in waiting-list mortality from $18 \%$ in the year before to $10 \%$ in the year after MELD was introduced $(P=$ 0.040) [4]. This result is in accordance with data already published on the 1-year mortality of patients on the waiting list for liver transplantation within the Eurotransplant area, comparing the period 2002-2005 (waiting-list mortality = $20 \%$ ) with the year 2007 (only 10\%) [5]. Other single-centre results within Europe have also confirmed a reduction in waiting-list mortality since the introduction of MELD [6]. Likewise, within the UNOS (United Network of Organ Sharing) area, where the MELD score was already implemented in February 2002, the introduction of MELD was followed by a steady decrease in waiting-list mortality and lower drop-out rates from waiting lists [7-9].

\section{MELD Score at Time of Organ Allocation}

After MELD implementation there was a significant increase in mean MELD score at the time of organ allocation, reflecting the intention to give priority to sicker patients on the waiting list. At our centre, the mean MELD score increased from 16.3 points in the year before to 22.4 points in the year after MELD introduction $(P=0.007)$ [4]. This observation accords with results from another single-centre analysis in Germany, where, again, a significant increase in mean MELD score at the time of liver transplantation, comparing the two years before and after MELD introduction, was observed (14.8 points versus 18.6 points; $P=0.001$ ) [10]. Since then, there has been a steady increase in mean MELD score within the Eurotransplant area and especially in Germany. For September 2010, a mean MELD score of 34 points for standard organ allocation (without standard exceptions, without high-urgency status) was reported for Germany [5]. This trend is also reflected in the Eurotransplant Annual Report 2010, which describes a $24 \%$ increase in the number of high-MELD recipients ( $\geq 30$ MELD points at the time of
TABLE 1: Standard MELD exceptions in Germany and their adjusted MELD score or adjusted 3-months-mortality, respectively [3].

\begin{tabular}{lc}
\hline Disease & $\begin{array}{c}\text { Initial match } \\
\text { MELD/adjusted } \\
\text { 3-month mortality }\end{array}$ \\
\hline Hepatocellular carcinoma (HCC) & $15 \%$ \\
Hepatoblastoma & MELD 30 \\
Polycystic liver disease & $10 \%$ \\
Hyperoxaluria type 1 & $10 \%$ \\
Persisting dysfunction following OLT & Current lab MELD plus \\
(including "small-for-size") & $20 \%$ \\
Cystic fibrosis & $10 \%$ \\
Familial amyloid polyneuropathy (FAP) & $15 \%$ \\
Hepatopulmonary syndrome & $15 \%$ \\
Portopulmonary hypertension (POPH) & $25 \%$ \\
Urea cycle disorders & MELD 30 \\
Morbus Osler & $15 \%$ \\
Hepatic epithelioid & $15 \%$ \\
hemangioendothelioma (HEHE) & \\
Biliary sepsis/secondary sclerosing & Current lab MELD plus \\
cholangitis (SSC) & $30 \%$ \\
Primary sclerosing cholangitis (PSC) & $15 \%$ \\
Cholangiocarcinoma & $10 \%$ \\
\hline
\end{tabular}

organ allocation) within the total population of liver-graft recipients in 2010 compared with 2009. In Germany in 2010, the proportion of high-MELD recipients among the total population of MELD-allocated liver transplantations was as high as $43 \%$ [11]. This might point to a specific problem in Germany, which has the highest proportion of high-MELD recipients compared with other Eurotransplant countries.

\section{Donor Graft Quality}

Since the adoption of MELD-based allocation there has been a significant increase in the numbers of donor grafts at our centre that fell within the scope of the extended donor criteria (EDC) as defined by the German Medical Association. In Germany, age distribution of organ donors showed a steadily increase of donors over 65 years during the last ten years. Finally, these donors accounted almost one-third of the organ donations in 2011 [12]. Hence, 30 percent of transplanted organs in 2011 already fulfilled EDC status only based on donor age irrespective of further medical details. As a consequence, the percentage of transplanted organs at our centre with deficiencies corresponding to at least one EDC increased from $24 \%$ in the year before to $60 \%$ in the year after the introduction of $\operatorname{MELD}(P=0.001)$. In fact, there was an eightfold increase in the number of transplanted organs with deficiencies corresponding to two EDCs $(P<0.05)[4]$. Retrospective validation of the donor risk index (DRI) [13] within the donor population in the Eurotransplant area between 2003 and 2007 showed that more than $50 \%$ of the transplanted organs had deficiencies corresponding to at least one EDC [14]. 


\section{Postoperative Survival}

Since the introduction of MELD-based allocation there have been several reports of impaired postoperative survival in Germany. The first publication describing reduced postoperative survival since MELD was a single-centre analysis, where the 90 -day survival was only $79.6 \%$ in the two years after MELD compared with $88.6 \%$ in the two years before $(P=0.03)[10]$. A German multicentre analysis including 462 patients confirmed the low 1-year survival rate of $75.8 \%$ one year after MELD. In particular, the group of high-MELD recipients ( $\geq 30$ points) showed only the very poor 1 -year survival rate of $53 \%$ [15]. At our centre, 90-day survival was found to remain stable at $90 \%$ when the year before and the two years after MELD were compared. There were also no significant differences in 1-year survival between different groups according to their MELD scores. The survival analysis showed a 1-year survival of $84 \%$ for the "MELD 6-19" group, compared with $81 \%$ for the "MELD 20-29" group and only $74 \%$ for the "MELD $\geq 30$ " group $(P=0.82)[4]$. A comprehensive analysis of UNOS data for the ten-year period from 1997 to 2006 showed stable rates of patient and graft survival even after the introduction of MELD in 2002 [8]. In the clinical context of living donation within the UNOS area, survival analysis showed a favourable outcome even for high-MELD recipients ( $\geq 30$ points), with a 1-year survival greater than $80 \%$ [16]. This might be due to the very strict patient selection that is practiced in connection with living donation. Therefore, we performed a retrospective analysis of our high-MELD recipients for factors predictive of impaired survival. This led us to the identification of the triad of dialysis, ventilatory support, and vasopressor administration within the 48 hours before liver transplantation as being highly predictive for reduced 1-year survival (30\% for the triad group versus $86 \%$ for the control group; $P<0.001$ ) [17].

\section{Discussion}

The implementation of MELD-based allocation has reflected a shift in allocation policy towards cases of medical urgency and has resulted in easier organ access for sicker patients, as reflected by a significant increase in mean MELD at time of organ allocation especially in Germany. In particular, a proportion of $43 \%$ high-MELD recipients with 30 or more MELD points represents the highest proportion compared to the most European countries. Although a comprehensive analysis of this development cannot be performed yet, there are several clinical factors which might contribute to this. On the one hand, the organ donor rate in Germany was only 15.8 per million population in 2010 , which is ranked in the lower third compared to other European countries [12]. On the other hand, there has been a significant increase in donor organs fulfilling extended donor criteria in Europe during the last decade. In Germany, 30 percent of transplanted organs in 2011 already fulfilled EDC status only based on donor age irrespective of further medical details. As a consequence, the limited number of organs suitable for liver transplantation in the sickest waiting list candidates with 30 or more MELD points is de facto reduced since EDC grafts are not optimal to pair with high-risk recipients. Thus, persisting donor organ shortage in Germany is becoming more aggravated since there is a growing discrepancy between the increasing number of severe ill transplant candidates as intended by the "sickest-first" concept and the limited number of donor grafts that do not meet EDC.

Through this intentional prioritization of sicker patients, MELD has clearly been effective in reducing waiting-list mortality. On the other hand, there have been several reports of impaired postoperative survival since introduction of MELD. According to these analyses, the major contributory factor to impaired overall survival is a significantly worse survival in the group of high-MELD recipients ( $\geq 30$ points) $[10,18,19]$. As a consequence, the possibility of imposing cut-off values for MELD score that might preclude transplantation because of increased postoperative mortality in these sicker patients has been discussed $[18,20]$.

However, comprehensive analyses have also been published that indicate no significant differences in survival between different MELD-score groups [4, 7, 21]. Furthermore, in the clinical context of living donation, favourable outcome in recipients even with 35 or more MELD points has been achieved [16, 22]. This emphasizes the importance of very strict patient selection, which is indispensable in the clinical setting of living donation. Nevertheless, as a consequence of the "sickest-first" concept implemented by the MELD allocation, the caveat must be made that it is probably not always possible to impose patient selection of a stringency comparable to that applied for living donation. Especially in high-MELD recipients, the decision on whether to accept an offered donor graft will often be difficult and confronts the physician with a dilemma of choice between hoping for a long-term advantage and avoiding an immediate risk. If a patient is in critical need of a liver transplant, and a donor graft of less than ideal quality is to hand, is it justified to postpone the transplant in the hope that a better organ might soon become available, while entailing the risk that it might not (with the consequent threat to the patient's short-term survival)?

Against the background of $43 \%$ high-MELD recipients among the total population of liver-graft recipients in Germany in the year 2010, close attention has to be paid to the analysis of risk factors for impaired survival in these severely ill patients [6]. Several approaches have been adopted to identify pre-operative parameters that predict postoperative outcome, in order to improve the allocation system in the sense of providing an equitable balance between medical urgency and ultimate outcome [23-25]. However, all these models seem to have their individual limitations [26]. At our centre, we follow strictly our simple clinical approach using the triad concept. With this policy we have been able to achieve a 1-year survival rate of $74 \%$ among our high-MELD recipients [17].

The triad concept is supported by recent published data from Munich on liver transplantation in patients with multiorgan failure $(n=18)$ [27]. Thirteen patients underwent transplantation directly from the Intensive Care Unit (ICU); ten of these 13 patients fulfilled the preoperative 
triad of dialysis, ventilatory support, and vasopressor administration. The 1-year survival was only $46 \%$ for these 13 patients from the ICU, which seems to be unacceptably poor. As a remarkable detail, the authors found that a decrease in MELD during the first 48 hours after admission to the ICU was associated with survival $(P=0.019)$. This finding also underlines the prognostic relevance of the first 48-hours interval after admission to the ICU. Clinical development during that period seems to determine whether substantial clinical amelioration can be achieved or whether the patient's clinical condition is in fact deteriorating, which in many cases would probably lead to exclusion from transplantation.

A further aspect that might have contributed to worsened survival since MELD implementation may have been the tendency to pair high-risk organs with lowest-risk patients (MELD 6-8 points). The increasingly ageing populationand consequently ageing donor organs_-raises the question of optimal donor-recipient matching [28]. Analysis of US data from the SRTR (Scientific Registry of Transplant Recipients) showed a significantly higher mortality in lowerMELD recipients matched with high-risk donor grafts, whereas all recipients with $\mathrm{MELD} \geq 20$ points had a significant benefit from transplantation even when receiving high-risk donor grafts [29]. Other data from the UNOS registry also showed worsened posttransplant survival in less urgent patients (MELD $<20$ points), which was primarily attributed to changes in donor-organ quality [30]. These data provide clear evidence for the benefit of organ utilization by matching high-risk donors with sicker patients on the waiting list (MELD 20-29 points). At our centre, we adhere to this matching procedure. Thus, recipients with a MELD score of 20-29 received significantly more often organs meeting at least one EDC [4].

\section{Conclusion}

MELD has been effective in the reduction of waiting-list mortality by giving priority to sicker patients on the waiting list. However, in Germany, impaired postoperative survival rates after MELD implementation have also been reported. Detailed analysis suggests that this is mainly attributable to significantly worse outcomes in the group of high-MELD recipients, which in 2010 represented $43 \%$ of all liver graft recipients in Germany. Therefore, an extensive multicentric analysis of risk factors for impaired survival in these severely ill patients appears indispensable. Continuous review and adaptation of the allocation system will remain a challenge.

\section{References}

[1] G. E. Jung, J. Encke, J. Schmidt, and A. Rahmel, "Model for end-stage liver disease: new basis of allocation for liver transplantations," Chirurg, vol. 79, no. 2, pp. 157-163, 2008.

[2] M. Malinchoc, P. S. Kamath, F. D. Gordon, C. J. Peine, J. Rank, and P. C. J. Ter Borg, "A model to predict poor survival in patients undergoing transjugular intrahepatic portosystemic shunts," Hepatology, vol. 31, no. 4, pp. 864-871, 2000.

[3] German guidelines for solid organ transplantation, 2011, http://www.bundesaerztekammer.de/.
[4] C. Benckert, M. Quante, A. Thelen et al., "Impact of the MELD allocation after its implementation in liver transplantation," Scandinavian Journal of Gastroenterology, vol. 46, no. 7-8, pp. 941-948, 2011.

[5] H. J. Schlitt, M. Loss, M. N. Scherer et al., "Current developments in liver transplantation in Germany: MELDbased organ allocation and incentives for transplant centres," Zeitschrift fur Gastroenterologie, vol. 49, no. 1, pp. 30-38, 2011.

[6] P. Dutkowski, C. E. Oberkofler, M. Béchir et al., "The model for end-stage liver disease allocation system for liver transplantation saves lives, but increases morbidity and cost: a prospective outcome analysis," Liver Transplantation, vol. 17, no. 6, pp. 674-684, 2011.

[7] R. B. Freeman Jr., D. E. Steffick, M. K. Guidinger, D. G. Farmer, C. L. Berg, and R. M. Merion, "Liver and intestine transplantation in the United States, 1997-2006," American Journal of Transplantation, vol. 8, no. 4, pp. 958-976, 2008.

[8] A. B. Massie, B. Caffo, S. E. Gentry et al., "MELD exceptions and rates of waiting list outcomes," American Journal of Transplantation, vol. 11, no. 11, pp. 2362-2371, 2011.

[9] H. J. Kim, J. J. Larson, Y. S. Lim et al., "Impact of MELD on waitlist outcome of retransplant candidates," American Journal of Transplantation, vol. 10, no. 12, pp. 2652-2657, 2010.

[10] T. J. Weismüller, A. Negm, T. Becker et al., "The introduction of MELD-based organ allocation impacts 3-month survival after liver transplantation by influencing pretransplant patient characteristics," Transplant International, vol. 22, no. 10, pp. 970-978, 2009.

[11] Eurotransplant Annual Report 2011, http://www.eurotransplant.org/cms/.

[12] DSO. Organ Donation and Transplantation in GermanyAnnual Report 2011, http://www.dso.de/.

[13] S. Feng, N. P. Goodrich, J. L. Bragg-Gresham et al., "Characteristics associated with liver graft failure: the concept of a donor risk index," American Journal of Transplantation, vol. 6, no. 4, pp. 783-790, 2006.

[14] J. J. Blok, A. E. Braat, R. Adam et al., "Validation of the donor risk index (DRI) in orthotopic liver transplantation within the eurotransplant region," Liver transplantation, vol. 18, no. 1, pp. 112-119, 2011.

[15] T. J. Weismüller, P. Fikatas, J. Schmidt et al., "Multicentric evaluation of model for end-stage liver disease-based allocation and survival after liver transplantation in Germanylimitations of the "sickest first"-concept," Transplant International, vol. 24, no. 1, pp. 91-99, 2011.

[16] S. Nadalin, R. Schaffer, and N. Fruehauf, "Split-liver transplantation in the high-MELD adult patient: are we being too cautious?" Transplant International, vol. 22, no. 7, pp. 702706, 2009.

[17] M. Quante, C. Benckert, and S. Jonas, [The preoperative triad of dialysis, ventilatory support and vasopressor administration is highly predictive for reduced survival following liver transplantation in high-MELD recipients] [Abstract, German] Current Congress, 2010.

[18] N. N. Onaca, M. F. Levy, E. Q. Sanchez et al., "A correlation between the pretransplantation MELD score and mortality in the first two years after liver transplantation," Liver Transplantation, vol. 9, no. 2, pp. 117-123, 2003.

[19] D. G. Maluf, E. B. Edwards, and H. M. Kauffman, "Utilization of extended donor criteria liver allograft: is the elevated risk of failure independent of the model for end-stage liver disease score of the recipient?" Transplantation, vol. 82, no. 12, pp. 1653-1657, 2006. 
[20] S. Habib, B. Berk, C. C. H. Chang et al., "MELD and prediction of post-liver transplantation survival," Liver Transplantation, vol. 12, no. 3, pp. 440-447, 2006.

[21] B. H. Ferraz-Neto, M. P. V. C. Zurstrassen, R. Hidalgo et al., "Analysis of liver transplantation outcome in patients with MELD score $\geq 30$," Transplantation Proceedings, vol. 40, no. 3 , pp. 797-799, 2008.

[22] M. Selzner, A. Kashfi, M. S. Cattral et al., "Live donor liver transplantation in high meld score recipients," Annals of Surgery, vol. 251, no. 1, pp. 153-157, 2010.

[23] A. Rana, M. A. Hardy, K. J. Halazun et al., "Survival outcomes following liver transplantation (SOFT) score: a novel method to predict patient survival following liver transplantation," American Journal of Transplantation, vol. 8, no. 12, pp. 25372546, 2008.

[24] W. R. Kim, S. W. Biggins, W. K. Kremers et al., "Hyponatremia and mortality among patients on the liver-transplant waiting list," The New England Journal of Medicine, vol. 359, no. 10, pp. 1018-1026, 2008.

[25] T. J. Weismüller, J. Prokein, T. Becker et al., "Prediction of survival after liver transplantation by pre-transplant parameters," Scandinavian Journal of Gastroenterology, vol. 43, no. 6, pp. 736-746, 2008.

[26] S. K. Asrani and W. R. Kim, "Organ allocation for chronic liver disease: model for end-stage liver disease and beyond," Current Opinion in Gastroenterology, vol. 26, no. 3, pp. 209-213, 2010.

[27] A. Umgelter, K. Lange, A. Kornberg, P. Buchler, H. Friess, and R. M. Schmid, "Orthotopic liver transplantation in critically ill cirrhotic patients with multi-organ failure: a single-center experience," Transplantation Proceedings, vol. 43, no. 10, pp. 3762-3768, 2011.

[28] R. Foster, M. Zimmerman, and J. F. Trotter, "Expanding donor options: marginal, living, and split donors," Clinics in Liver Disease, vol. 11, no. 2, pp. 417-429, 2007.

[29] D. E. Schaubel, C. S. Sima, N. P. Goodrich, S. Feng, and R. M. Merion, "The survival benefit of deceased donor liver transplantation as a function of candidate disease severity and donor quality," American Journal of Transplantation, vol. 8, no. 2, pp. 419-425, 2008.

[30] M. L. Volk, A. S. F. Lok, S. J. Pelletier, P. A. Ubel, and R. A. Hayward, "Impact of the model for end-stage liver disease allocation policy on the use of high-risk organs for liver transplantation," Gastroenterology, vol. 135, no. 5, pp. 15681574, 2008. 


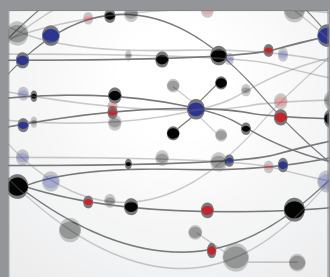

The Scientific World Journal
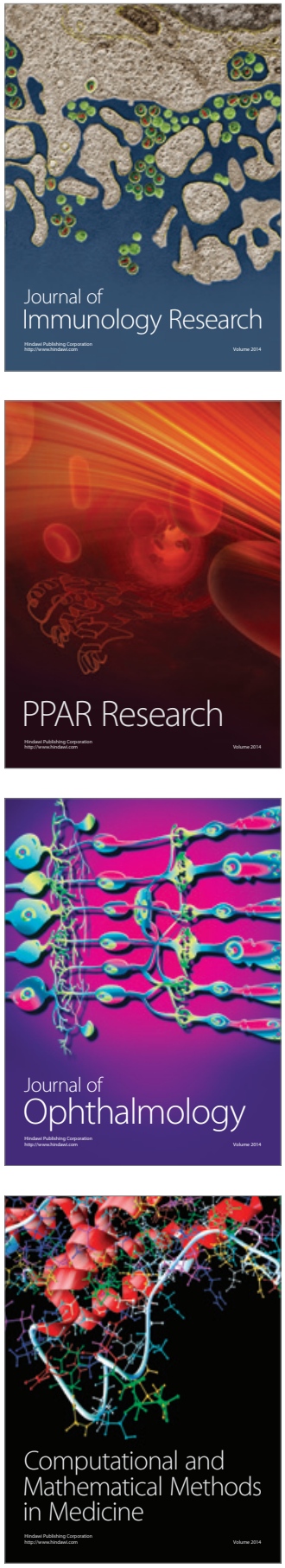

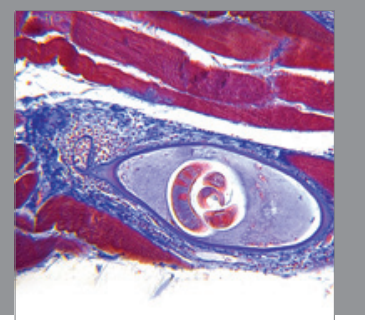

Gastroenterology

Research and Practice
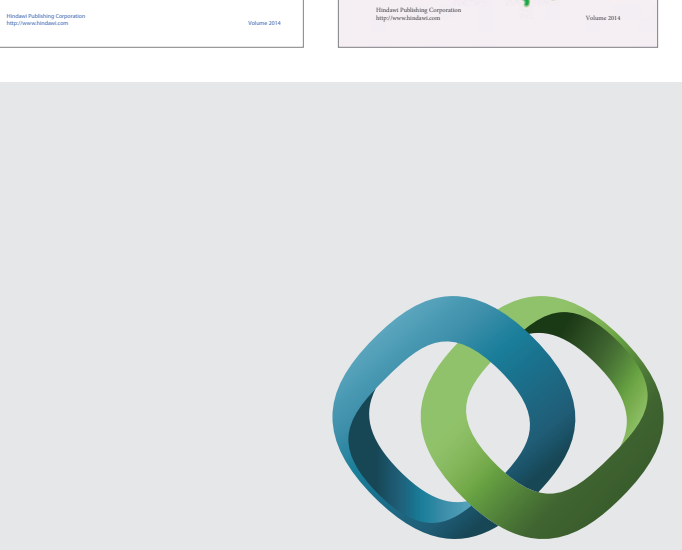

\section{Hindawi}

Submit your manuscripts at

http://www.hindawi.com
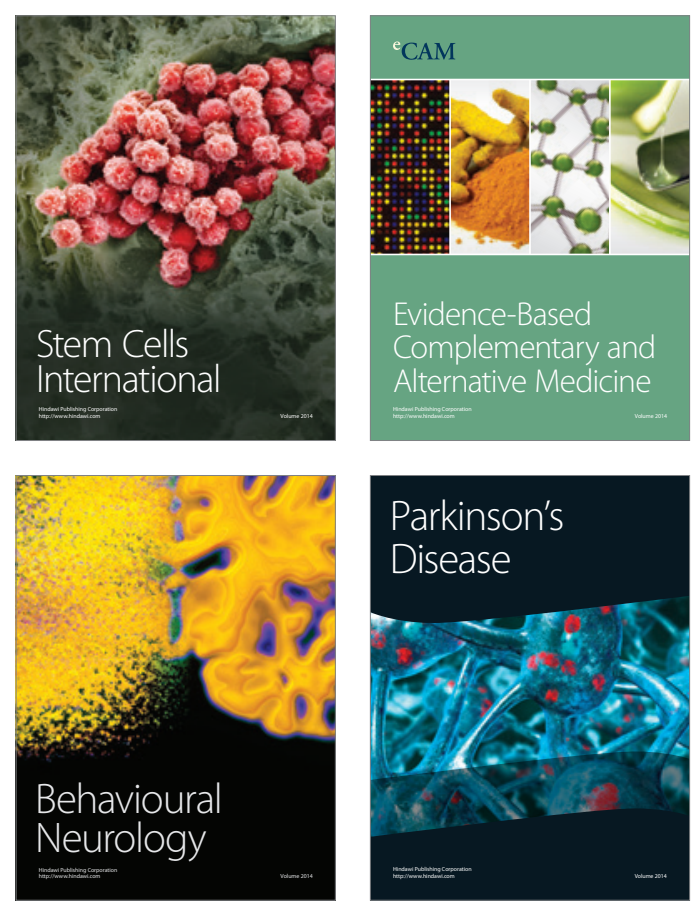

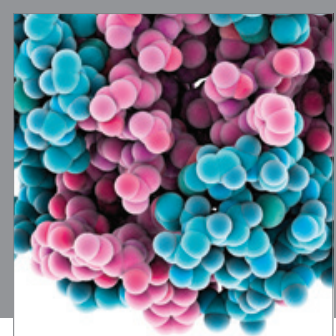

Journal of
Diabetes Research

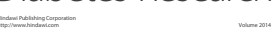

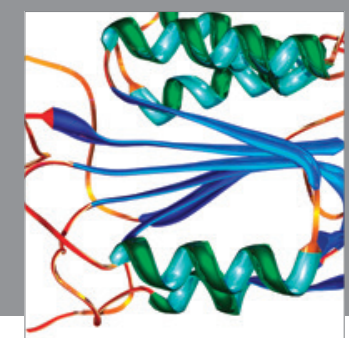

Disease Markers
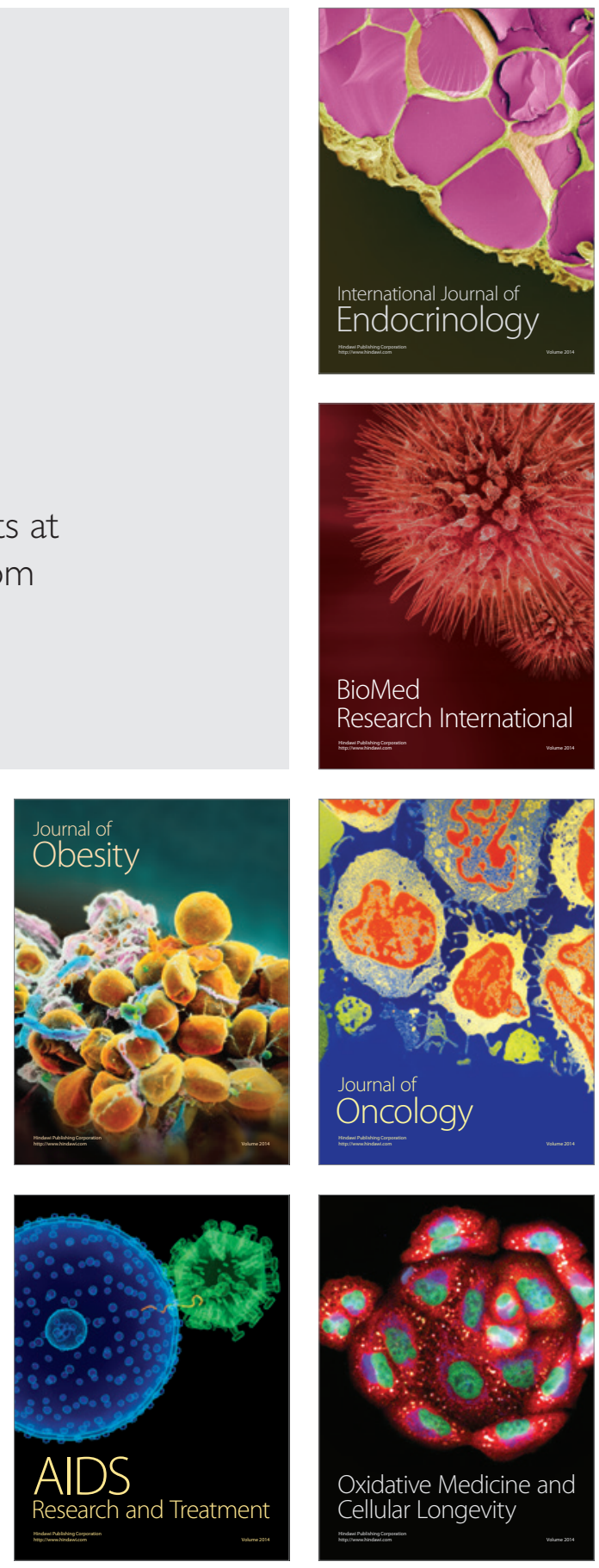